\title{
HEAVY QUARK CONFINEMENT FROM DYSON-SCHWINGER EQUATIONS
}

\author{
Conrad Burden \\ Department of Theoretical Physics, \\ Research School of Physical Sciences and Engineering, \\ Australian National University, Canberra ACT 0200, AUSTRALIA
}

\begin{abstract}
A formalism for studying the confinement of heavy quarks by considering the renormalised quark Dyson-Schwinger equation in the limit $m_{Q} \rightarrow \infty$ is described. We are particularly interested in studying the analytic structure of heavy quark propagators in the region of the complex momentum plane close to the bare fermion mass pole.
\end{abstract}

\section{Introduction}

Confinement and chiral symmetry breaking in quantum chromodynamics (QCD) must ultimately have a field theoretic cause. They are innately nonperturbative phenomena, demanding the use of appropriate techniques if they are to be fully understood. Lattice gauge theory and the Dyson-Schwinger (DS) equation techniqued represent the two most promising lines of attack on QCD from a nonperturbative, field theoretic point of view.

Of these two, lattice gauge theory has the advantage that it is as close as one can find to an $a b$ initio treatment of QCD. Approximations are clearly defined and in principle controllable. It has the disadvantage that a qualitative understanding of physical phenomena tends to be obscured by numerical brute force. Some progress is now being made, however, in interpreting lattice gauge fields in terms of monopoles and flux tubes.2

Dyson-Schwinger equations provide a complementary approach to QCD. The infinite tower of coupled integral equations relating Greens functions is an exact, nonperturbative description of QCD. Before any progress can be made, the tower must be truncated, generally at the level of quark and gluon propagator equations and bound state Bethe-Salpeter and Faddeev equations. Chiral symmetry breaking is signalled by the generation of a non-zero scalar part to the quark propagator, while a sufficient condition for quark confinement is the absence of real, timelike singularities in the propagator. These conditions ensue provided the dressed gluon propagator is sufficiently infrared enhanced. 3

Numerical solution of the gluon propagator DS equation is a formidable task, and opinions differ about the resultant infrared behaviour of the gluon propagator. 
been argued however that, if the three gluon and ghost-gluon vertices are modelled in a way free of singularities, a strongly infrared enhanced gluon propagator may result, leading to the required chiral symmetry breaking and quark confinement.6 Within the DS picture, these two phenomena go hand in hand, both driven by the functional form of the quark propagator.

There is of course no necessary inconsistency between the DS picture and dual superconductor flux tube models. A strongly infrared enhanced gluon propagator can readily generate a Wilson area law A fortiori, one should not be perturbed that the flux tube picture is not immediately manifest in the interpretation of a timelike singularity free propagator as a signal of confinement. After all, that Wilson loops should satisfy an area law (which we interpret as the existence of a flux tube) is not an immediate, or even necessary consequence of the fact that Polyakov loops are zero valued (which we interpret as a signal of colour confinement). Obviously, both pictures of confinement can happily coexist.

In what follows we give a very brief description of a formalism designed to extend the DS picture to the realm of heavy quarks 80 We shall see that the DS picture of quark confinement can be carried over to the extreme heavy quark limit in a natural and instructive way.

\section{The Heavy Quark Limit}

Our starting point is the quark DS equation

$$
\begin{aligned}
S(p)^{-1} & =i \gamma \cdot p A\left(p^{2}\right)+B\left(p^{2}\right) \\
& =Z_{2}\left[\gamma \cdot p+m_{0}(\Lambda)\right]+Z_{1} \int^{\Lambda} \frac{d^{4} q}{(2 \pi)^{4}} g^{2} D_{\mu \nu} \gamma_{\mu} S(q) \Gamma_{\nu}(q, p)
\end{aligned}
$$

with $S, D_{\mu \nu}$ and $\Gamma_{\mu}$ the renormalised dressed quark propagator, gluon propagator and proper quark-gluon vertex respectively. The renormalisation scale $\mu$ is set such that $\left.S(p)\right|_{p^{2}=\mu^{2}}=1 /\left[i \gamma \cdot p+m_{R}\left(\mu^{2}\right)\right]$. We use a Euclidean metric in which timelike vectors satisfy $p^{2}=-p_{\text {Minkowski }}^{2}<0$, and for which $\left\{\gamma_{\mu}, \gamma_{\nu}\right\}=2 \delta_{\mu \nu}$.

The heavy quark limit is taken by assuming the expansions

$$
A\left(p^{2}, \mu^{2}\right)=1+\frac{\Sigma_{A}(K, \kappa)}{m_{R}\left(\mu^{2}\right)}+\ldots, \quad B\left(p^{2}, \mu^{2}\right)=m_{R}\left(\mu^{2}\right)+\Sigma_{B}(K, \kappa)+\ldots,
$$

where we have defined the momentum variable $K$ and renormalisation point $\kappa$ by

$$
K=\frac{p^{2}+m_{R}^{2}}{2 i m_{R}}, \quad \kappa=\frac{\mu^{2}+m_{R}^{2}}{2 i m_{R}} .
$$

The change of independent variable $p^{2} \rightarrow K$ induced by the transformation Eq. (4) is discussed in detail in reference 8 . The main point to note here is that the real timelike $p^{2}$ axis in the vicinity of the 'would-be bare fermion mass pole', $p^{2}=-m^{2}$, becomes the imaginary $K$ axis in the new coordinates. The aim of the exercise is to solve for the quark propagator in the vicinity of the bare mass pole $K=0$. 


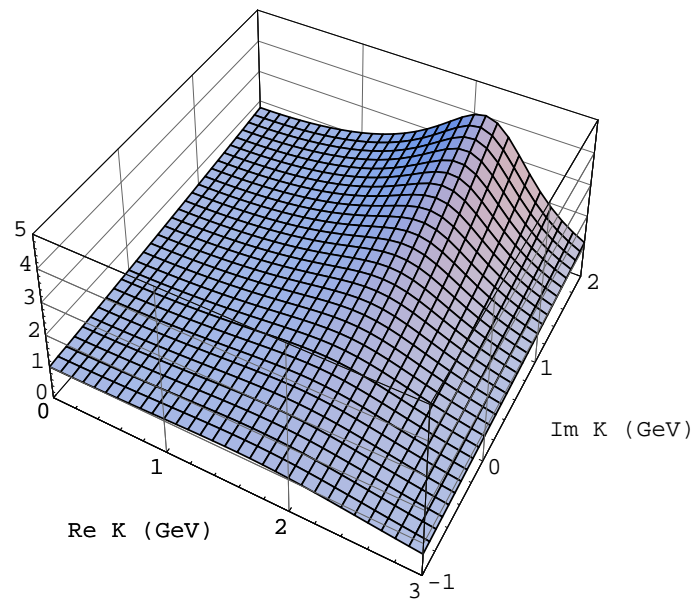

Fig. 1. The modulus $\left|\sigma_{Q}(K)\right|$ of the heavy quark propagator in the complex $K$ plane obtained by solving the heavy quark DS equation in bare vertex approximation with the gluon propagator $\Delta\left(k^{2}\right)$.

For the purposes of expanding the DS equation in the inverse heavy quark mass we introduce the momentum substitutions generic to heavy quark effective theory,

$$
p_{\mu}=i m_{R} v_{\mu}+k_{\mu}
$$

where $v_{\mu} v_{\mu}=1$. This gives $K=k \cdot v+k^{2} /\left(2 i m_{R}\right)$, and so in the vicinity of $k_{\mu}=0$, or $K=0$, the dressed quark propagator has the form,

$$
\begin{aligned}
S(p, \mu) & =\frac{1+\gamma \cdot v}{2} \frac{1}{i K+\Sigma_{B}(K, \kappa)-\Sigma_{A}(K, \kappa)}+O\left(\frac{1}{m_{R}}\right) \\
& \stackrel{\text { def }}{=} \frac{1+\gamma \cdot v}{2} \sigma_{Q}(K, \kappa)+O\left(\frac{1}{m_{R}}\right) .
\end{aligned}
$$

The confinement criterion that $S(p)$ should be free from timelike singularities on the negative real $p^{2}$ axis translates in the heavy quark case to a requirement that $\sigma_{Q}$ should be free from singularities on the imaginary $K$ axis.

In reference 10 the heavy quark propagator is solved for using the bare vertex or "rainbow" approximation $\Gamma_{\mu}(q, p)=\gamma_{\mu}$, and Landau gauge smeared Frank and 
Roberts 11 model gluon propagator $D_{\mu \nu}(k)=\left(\delta_{\mu \nu}-k_{\mu} k_{\nu} / k^{2}\right) \Delta\left(k^{2}\right)$, where

$$
\Delta\left(k^{2}\right)=(2 \pi)^{4} \frac{m_{t}^{2} d}{\alpha^{2} \pi^{2}} e^{-k^{2} / \alpha}+4 \pi^{2} d \frac{1-e^{-k^{2} /\left(4 m_{t}^{2}\right)}}{k^{2}} .
$$

Here $d=12 /\left(33-2 N_{f}\right), N_{f}=3$ is the number of light quark flavours, $m_{t}=0.69 \mathrm{GeV}$ is a parameter fitted to a range of calculated pion observables, and the Gaussian width $\alpha$ is chosen to be $0.5643(\mathrm{GeV})^{2}$.

In Fig. 1 is plotted the modulus $\left|\sigma_{Q}(K)\right|$ of the heavy quark propagator obtained by solving the DS equation in the vicinity of $K=0$, with a renormalised heavy quark mass of $m_{R}=5 \mathrm{GeV}$. We see that the bare propagator pole at $K=0$ has moved well away from the imaginary $K$ axis, leaving the heavy quark propagator analytic over a large region in the vicinity of $K=0$. The removal of propagator poles from the imaginary $K$ axis signals heavy quark confinement. As noted in reference $\mathrm{\theta}$, a large shift of the pole portends well for the possibility of finding solutions to the Bethe-Salpeter equation for $Q-\bar{q}$ mesons. 8 Solution of the heavy meson Bethe-Salpeter equation, of which accurate determination of heavy quark propagators is a necessary first step, should prove helpful in impulse approximation studies of semileptonic decays.12

\section{Acknowledgement}

The author acknowledges helpful discussions with F. T. Hawes and C. D. Roberts, and the hospitality of the Special Research Centre for the Subatomic Structure of Matter in Adelaide for hosting the Workshop on Nonperturbative Methods in Quantum Field Theory where part of this work was completed.

\section{References}

1. C. D. Roberts and A. G. Williams, Prog. Part. and Nucl. Phys. 33, 475 (1994).

2. G. Bali, The Mechanism of Quark Confinement, these proceedings.

3. C. D. Roberts, "Nonperturbative QCD with Modern Tools", nuclth/9807026.

4. M. R. Pennington, "Calculating hadronic properties in strong QCD", hepph/9611242.

5. D. Atkinson and J. C. R. Bloch, Mod. Phys. Lett. A 13, 1055 (1998), and references therein.

6. F. T. Hawes, P. Maris and C. D. Roberts, "Infrared behaviour of propagators and vertices", nucl-th/9807056.

7. G. B. West, Phys. Lett. B115, 468 (1982).

8. C. J. Burden and D.-S. Liu, Phys. Rev. D 55, 367 (1997).

9. C. J. Burden, Phys. Rev. D 57, 276 (1998).

10. C. J. Burden, "The effect of the ultraviolet part of the gluon propagator on the heavy quark propagator", hep-ph/9807438.

11. M. R. Frank and C. D. Roberts, Phys. Rev. C 53, 390 (1996).

12. M. A. Ivanov, Yu. L. Kalinovsky, P. Maris and C. D. Roberts, Phys. Lett. B 416, 29 (1998); Phys. Rev. C 57, 1991 (1998). 